\title{
Ab Initio Study of the Early Stage of Si Epitaxy on the Chlorinated Si(100) Surface
}

\author{
Tatiana V. Pavlova ${ }^{1,2}$ * Egor S. Skorokhodov ${ }^{1,3}$, Georgy M. Zhidomirov ${ }^{1,2}$, and Konstantin N. Eltsov ${ }^{1,2}$ \\ ${ }^{1}$ Prokhorov General Physics Institute of the Russian Academy of Sciences, Moscow, Russia \\ ${ }^{2}$ National Research University Higher School of Economics, Moscow, Russia and \\ ${ }^{3}$ Moscow Institute of Physics and Technology, Dolgoprudny, Russia
}

\begin{abstract}
The homoepitaxial growth of $\mathrm{Si}$ on $\mathrm{Si}(100)$ covered by a resist mask is a necessary technological step for the fabrication of donor-based quantum devices with scanning tunneling microscope lithography. In the present work, the chlorine monolayer is selected as the resist. Using density functional theory, we investigated the adsorption of a single silicon atom on $\mathrm{Si}(100)-2 \times 1-\mathrm{Cl}$ as the starting process of $\mathrm{Si}$ epitaxy. The incorporation of a silicon atom under a $\mathrm{Cl}$ monolayer proved to be the most energetically favorable process. Our results show that chlorine segregates on the surface during $\mathrm{Si}$ deposition and does not incorporate into homoepitaxial layers. In addition, we found that $\mathrm{SiCl}_{2}^{*}$, $\mathrm{SiCl}_{3}^{*}$, and $\mathrm{SiCl}_{4}^{*}$ clusters can be formed above a $\mathrm{Si}(100)-2 \times 1-\mathrm{Cl}$ surface while $\mathrm{Si}$ is adsorbed. $\mathrm{SiCl}_{2}^{*}$ clusters are bound weakly to the substrate, and their desorption leaves the silicon surface free of chlorine. To check whether the Si epitaxy is possible on the chlorine resist, we compare our results with the well-studied case of a hydrogen resist. We find the two processes to be similar; moreover, epitaxy on chlorine resist appears to have an advantage.
\end{abstract}

\section{INTRODUCTION}

Homoepitaxy of silicon is widely utilized for dopant encapsulation in atomic scale devices. A sufficiently thick crystalline silicon layer (about $50 \mathrm{~nm}$ ) should be grown on top of a surface with dopants to preserve the electronic properties of semiconducting devices from unwanted surface effects. Silicon epitaxy is a necessary step for the fabrication of $\delta$-layers 1,2 , nanowires 3.4 , and quantum $\operatorname{dots}^{5}$. In addition, high quality of epitaxial layers is extremely important for phosphorus-in-silicon quantum computer building blocks6.7.

To create the desired two-dimensional structure of impurities, a silicon surface is covered with a resist which is then patterned (locally removed) with a scanning tunneling microscope (STM) tip. After the adsorption of molecules containing specific impurities, impurity atoms are embedded on the resist-free sites and then the surface is covered with epitaxial silicon layers. The quality of epitaxial layers strongly depends on the interaction of $\mathrm{Si}$ adatoms with atoms of the resist used for mask fabrication. If a hydrogen monolayer is used as a mask for the patterning of $\mathrm{Si}(100)-2 \times 1$ surface, epitaxial layers of good quality are obtained by silicon overgrowth at $250^{\circ} \mathrm{C}^{6,8,9}$, but dopant diffusion is not negligible ${ }^{9}$. However, to control the positions of the dopants precisely, it is necessary to prevent the movement of the $\mathrm{P}$ donors during silicon overgrowth. Although dopant diffusion can be minimized by growing several locking layers at room temperature prior to higher-temperature Si epitaxy ${ }^{2,9,10}$, this partially suppresses the electrical activation of the dopants 2,9 . There is a challenge in looking for an optimal solution, retaining the dopants in their original embedded places without suppressing their activation.

Theoretical calculations of Si atom adsorption and diffusion on H-terminated $\mathrm{Si}(100)-2 \times 1$ surface have provided an insight into the mechanism of homoepitaxy ${ }^{11-13}$. A silicon atom adsorbed on $\mathrm{Si}(100)-2 \times 1-\mathrm{H}$ can spontaneously substitute a hydrogen atom on the surface and then forms Si dihydride. Experimental results confirm the presence of surface Si dihydrides at submonolayer coverages $^{14}$. As such a Si film grows, most $\mathrm{H}$ atoms segregate on the surface and are not incorporated into the epitaxial film ${ }^{8,15}$.

A chlorine monolayer on a silicon surface can also be utilized as a resist ${ }^{16,17}$. The key difference between a hydrogen and chlorine resist is the potential possibility to remove substrate atoms $(\mathrm{Si})$ together with resist (Cl) atoms by the STM tip because of the strong interaction of chlorine with silicon. This claim is supported by a well-known etching effect that chlorine demonstrates on $\mathrm{Si}(100)$, studied both theoretically 18 and experimentally ${ }^{19}$. Of particular interest is a proposal to use STM lithography on $\mathrm{Si}(100)-2 \times 1-\mathrm{Cl}$ for placing $\mathrm{P}$ atoms with atomic precision ${ }^{20}$. After positioning a $\mathrm{P}$ atom on a patterned Si surface, we have to build the silicon lattice with an additional Si layer of $30-50 \mathrm{~nm}$ as for $\mathrm{Si}(100)-2 \times 1-\mathrm{H}$ case. Si homoepitaxy directly on a chlorinated Si surface looks a reasonable way to do it. While the interaction of chlorosilanes and $\mathrm{HCl}$ with silicon surface was investigated to find the mechanism of silicon epitaxy ${ }^{21}-23$, and the interaction of various molecules with chlorinated silicon surfaces was considered for the development of functionalized surfaces 24,25 and for the investigation of the reactivity of chlorinated silicon surface 26,27 , there are no theoretical or experimental studies of the adsorption of silicon atoms on a Cl-terminated $\mathrm{Si}(100)-2 \times 1$ surface.

In this paper, we report the results of density functional theory (DFT) calculations of Si atom adsorption on $\mathrm{Si}(100)-2 \times 1-\mathrm{Cl}$. A silicon adatom spontaneously substitutes a $\mathrm{Cl}$ atom and further migrates to the most stable inter-bridge dimer site (bound with two Si and two $\mathrm{Cl}$ atoms). This process is similar to that in the case of $\mathrm{Si}(100)-2 \times 1-\mathrm{H}$ surface, and should lead to $\mathrm{Cl}$ atoms segregation during $\mathrm{Si}$ epitaxy. Despite the small radius of hydrogen being suggested 12 as a reason for spontaneous substitutional adsorption of silicon on $\mathrm{Si}(100)-2 \times 1-\mathrm{H}$, the 
same adsorption mechanism is found to work for a chlorinated silicon surface. Moreover, there is an additional pathway of spontaneous Si adsorption with formation of $\mathrm{SiCl}_{2}^{*}$ clusters weakly bound with the silicon surface. Desorption of $\mathrm{SiCl}_{2}^{*}$ clusters from the surface requires low activation energy, so chlorine can be removed without annealing.

\section{CALCULATION METHOD}

First-principle calculations of silicon atom adsorption on $\mathrm{Si}(100)-2 \times 1-\mathrm{Cl}$ surface were performed with spin-polarized density functional theory implemented in VASP 28,29 . The generalized gradient approximation with the exchange-correlation functional in the form of Perdew-Burke-Ernzerhof (PBE) was applied ${ }^{30}$. The eigenfunctions of valence electrons were expanded in a plane waves basis set with an energy cutoff of $350 \mathrm{eV}$. The $\operatorname{Si}(100)$ surface was simulated by recurring $4 \times 4$ cells, each consisting of eight atomic layers of silicon. The slabs were separated by vacuum gaps of approximately $15 \AA$. The bottom three layers were fixed at bulk positions, while the other silicon layers were allowed to relax. The lowest layer was covered by hydrogen atoms to saturate the dangling bonds of silicon. Chlorine atoms were placed on the upper side of the slab to form a $\mathrm{Si}(100)-2 \times 1-\mathrm{Cl}$ structure. Reciprocal cell integrations were performed using the $4 \times 4 \times 1 \mathrm{k}$-points grid.

The adsorption energy $\left(E_{a d s}\right)$ of a silicon adatom was calculated as the difference between the total energy of the surface with the adatom $\left(E_{S i+s u r f}\right)$ and the total energies of the $\mathrm{Si}(100)-2 \times 1-\mathrm{Cl}$ surface $\left(E_{\text {surf }}\right)$ and a $\mathrm{Si}$ atom in the gaseous phase $\left(E_{S i}\right)$ :

$$
E_{a d s}=E_{S i+s u r f}-E_{s u r f}-E_{S i} .
$$

The activation barriers $\left(E_{\text {act }}\right)$ were calculated using the climbing nudged-elastic band (CI-NEB) method 31 with six images (including the two end points).

\section{RESULTS}

Figure 1 shows the obtained adsorption structures for $\mathrm{Si}$ adatom $\left(\mathrm{Si}_{a d}\right)$ on a $\mathrm{Si}(100)-2 \times 1-\mathrm{Cl}$ surface. There are two types of adsorption structures: $\mathrm{Si}_{a d}$ bound with $\mathrm{Cl}$ atoms (type I) and $\mathrm{Si}_{a d}$ bound with $\mathrm{Si}$ surface atom(s) $\left(\mathrm{Si}_{s}\right)$ (type $\left.\mathrm{II}\right)$. The structures of type II are more energetically favorable, therefore the formation of $\mathrm{Si}_{a d}-\mathrm{Si}_{s}$ bonds stabilizes the surface structure. We discuss further only one structure of type I for each kind of bonds: structure $\mathrm{A}$ (two $\mathrm{Si}_{a d}-\mathrm{Cl}$ bonds, all $\mathrm{Cl}$ atoms are bound with the surface), structure $\mathrm{B}$ (two $\mathrm{Si}_{a d}-\mathrm{Cl}$ bonds, only one $\mathrm{Cl}$ atom is bound with the surface), structure $\mathrm{C}$ (three $\mathrm{Si}_{a d}-\mathrm{Cl}$ bonds), and structure $\mathrm{D}$ (four $\mathrm{Si}_{a d}-\mathrm{Cl}$ bonds). Structural parameters for all structures are summarized in Table 1
Type I. $\mathrm{Si}_{\mathrm{ad}}-\mathrm{Cl}$ bond(s)
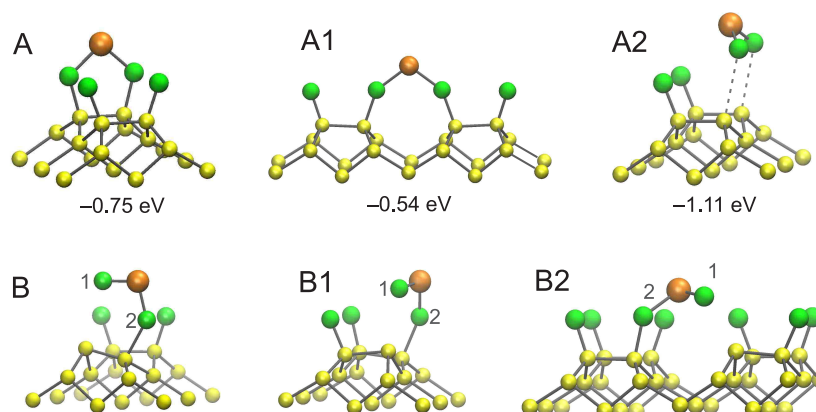

$-1.51 \mathrm{eV}$
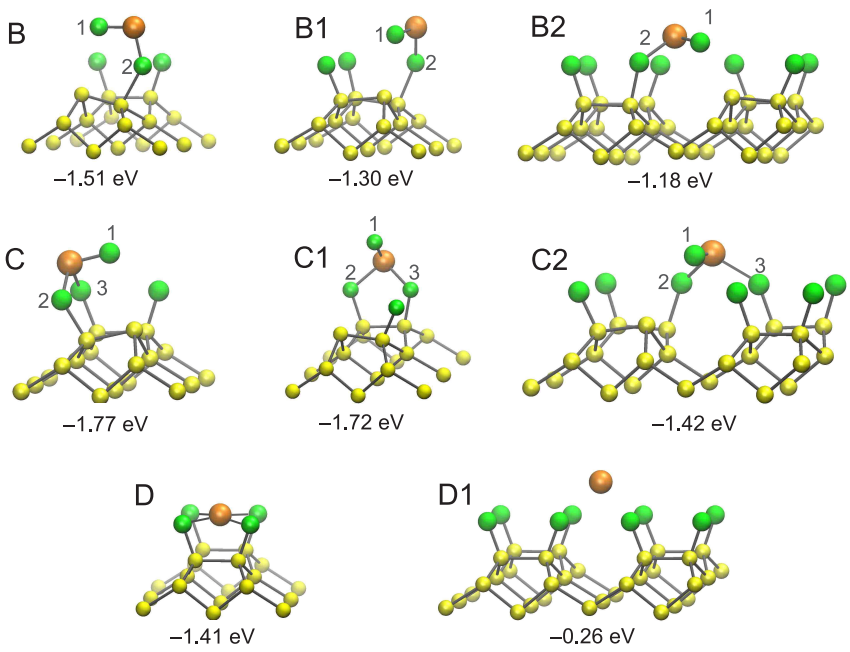

Type II. $\mathrm{Si}_{\mathrm{ad}}-\mathrm{Si}_{\mathrm{s}}$ bond(s)

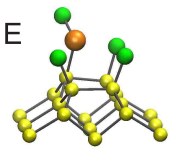

$-2.83 \mathrm{eV}$

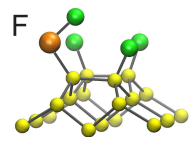

$-2.89 \mathrm{eV}$

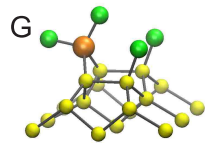

$-3.44 \mathrm{eV}$

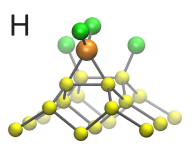

$-3.24 \mathrm{eV}$
Figure 1. Optimized atomic structures for $\mathrm{Si}_{a d}$ adsorbed on Si(100)-2×1-Cl. Adsorption energies are indicated for every structure. Silicon atoms are yellow, chlorine atoms are green, and $\mathrm{Si}$ adatom is orange.

In the structure $\mathrm{A}$, a $\mathrm{SiCl}_{2}^{*}$ cluster is formed (asterisk denotes a cluster attached to the surface via $\mathrm{Cl}$ atom(s)). In the cluster, the Si adatom forms two equivalent bonds with $\mathrm{Cl}$ atoms belonging to the same silicon dimer. Structural parameters of the resulting $\mathrm{SiCl}_{2}^{*}$ cluster appear to be equal to those calculated for the $\mathrm{SiCl}_{2}$ molecule in vacuum $\left(\mathrm{Si}-\mathrm{Cl}\right.$ bond length $=2.09 \AA, \angle\left(\mathrm{Cl}-\mathrm{Si}_{a d}-\mathrm{Cl}\right)=$ $\left.102^{\circ}\right)$.

In the structure $\mathrm{B}$, one chlorine atom of $\mathrm{SiCl}_{2}^{*}$ cluster forms a stronger bond with the surface, therefore the cluster structure is slightly different from the free $\mathrm{SiCl}_{2}$ molecule. $\mathrm{SiCl}_{2}^{*}$ cluster can also be formed with $\mathrm{Cl}$ belonging to different silicon dimers (structure B2).

$\mathrm{SiCl}_{3}^{*}$ and $\mathrm{SiCl}_{4}^{*}$ clusters formation is also possible (structures $\mathrm{C}$ and $\mathrm{D}$, correspondingly). The $\mathrm{SiCl}_{3}^{*}$ cluster is the most stable one among other $\operatorname{SiCl}_{x}^{*}(x=2,3,4)$ clusters attached to the surface via $\mathrm{Cl}$ atom(s).

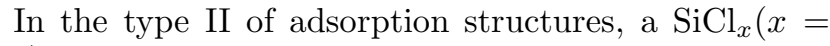
$1,2)$ cluster is attached to the surface via $\mathrm{Si}_{a d}-\mathrm{Si}_{s}$ 
Table I. Adsorption energies and lattice parameters for the structures shown in Fig. 1 bond length between Si adatom $\left(\mathrm{Si}_{a d}\right)$ and the nearest $\mathrm{Cl}$ atom(s); bond length between $\mathrm{Si}$ surface atom $\left(\mathrm{Si}_{s}\right)$ and $\mathrm{Cl}$ atom of cluster formed by $\mathrm{Si}_{a d}$; bond length between $\mathrm{Si}_{a d}$ and the nearest $\mathrm{Si}_{s}$; and angle between $\mathrm{Si}_{a d}$ and the nearest $\mathrm{Cl}$ atoms of the cluster.

\begin{tabular}{|c|c|c|c|c|c|}
\hline Struct. & $E_{a d s}, \mathrm{eV}$ & $\mathrm{Si}_{a d}-\mathrm{Cl}, \AA$ & $\mathrm{Si}_{s}-\mathrm{Cl}, \AA$ & $\mathrm{Si}_{a d}-\mathrm{Si}_{s}, \AA$ & $\angle\left(\mathrm{Cl}-\mathrm{Si}_{a d^{-}} \mathrm{Cl}\right)$ \\
\hline $\mathrm{A}$ & -0.75 & 2.36 & 2.25 & 3.94 & $96^{\circ}$ \\
\hline $\mathrm{A} 1$ & -0.54 & 2.38 & 2.28 & 4.45 & $107^{\circ}$ \\
\hline $\mathrm{A} 2$ & -1.11 & 2.10 & 3.75 & 5.10 & $102^{\circ}$ \\
\hline $\mathrm{B}$ & -1.51 & $\mathrm{Cl}(1): 2.06$ & 3.89 & 4.41 & $104^{\circ}$ \\
& & $\mathrm{Cl}(2): 2.18$ & 2.57 & & \\
\hline $\mathrm{B} 1$ & -1.30 & $\mathrm{Cl}(1): 2.04$ & 3.59 & 4.48 & $103^{\circ}$ \\
& & $\mathrm{Cl}(2): 2.21$ & 2.48 & & \\
\hline $\mathrm{B} 2$ & -1.18 & $\mathrm{Cl}(1): 2.04$ & 3.24 & 4.26 & $96^{\circ}$ \\
& & $\mathrm{Cl}(2): 2.42$ & 2.26 & & \\
\hline $\mathrm{C}$ & -1.77 & $\mathrm{Cl}(1): 2.07$ & 4.18 & 4.16 & $99^{\circ}$ \\
& & $\mathrm{Cl}(2): 2.35$ & 2.33 & & \\
\hline $\mathrm{C} 1$ & -1.72 & $\mathrm{Cl}(1): 2.55$ & 2.20 & & $90-95^{\circ}$ \\
& & $\mathrm{Cl}(2): 2.46$ & 2.17 & 3.86 & 18,19 \\
\hline $\mathrm{C} 2$ & -1.42 & $\mathrm{Cl}(1): 2.05$ & 4.06 & 3.86 & $99-103^{\circ}$ \\
& & $\mathrm{Cl}(2): 2.33$ & 2.33 & & \\
\hline $\mathrm{D}$ & -1.41 & 2.70 & 2.14 & 3.18 & $89-91^{\circ}$ \\
\hline $\mathrm{D} 1$ & -0.26 & 3.20 & 2.09 & 5.10 & $69-70^{\circ}$ \\
\hline $\mathrm{E}$ & -2.83 & 2.09 & 3.68 & 2.50 & - \\
\hline $\mathrm{F}$ & -2.89 & 2.09 & 3.57 & 2.50 & - \\
\hline $\mathrm{G}$ & -3.44 & 2.05 & 3.91 & 2.44 & $106^{\circ}$ \\
\hline $\mathrm{H}$ & -3.24 & 2.05 & 3.84 & 2.36 & $106^{\circ}$ \\
\hline
\end{tabular}

bond(s) (such clusters are denoted without asterisk). In the structure E (Fig. 1), Si adatom is bound with the silicon lattice, and the chlorine segregates on top of the surface structure. To obtain the structure E, the structure with a $\mathrm{Si}$ adatom at a distance of $3.5 \AA$ above the chlorine monolayer was optimized using VASP code. The lateral position of $\mathrm{Si}_{a d}$ was shifted from the top position of the $\mathrm{Cl}$ atom in the directions [110] (towards the dimer) and [110] by $0.50 \AA$. Figure 2 shows the initial structure (Fig. 2a), the three structures through which the process of coordinates relaxation passes (Fig. 20 b-d), and the optimized structure E (Fig. 2e). (From the set of structures, we have chosen the three structures in which the formation of bonds between the silicon atom and the neighboring chlorine atoms is most clearly seen.) At the beginning (Fig. 20), the $\mathrm{Si}$ adatom is attracted to the nearest chlorine atom $\mathrm{Cl}(1)$, and the bond between chlorine $\mathrm{Cl}(1)$ and the substrate $\mathrm{Si}$ atom becomes weaker. Then, Si adatom exchanges places with chlorine $\mathrm{Cl}(1)$ and forms bonds with the nearest chlorine atoms $\mathrm{Cl}(2)$ and $\mathrm{Cl}(3)$ (Fig. 22,d). The process of exchanging chlorine and silicon atoms occurs spontaneously. We believe that the energy required for $\mathrm{Cl}(1)-\mathrm{Si}_{s}$ bond breaking is compensated by the creation of bonds between $\mathrm{Si}_{a d}$ and the neighboring chlorine atoms $(\mathrm{Cl}(2)$ and $\mathrm{Cl}(3))$. In the final position (Fig. 2 2 ), the bond length between $\mathrm{Si}$ adatom and the nearest $\mathrm{Si}$ atom is slightly longer than that in the bulk $(2.50 \AA$ vs $2.37 \AA)$. It is worth mentioning, substitutional adsorption leads to the most energetically favorable structure $\left(E_{a d s}=-2.83 \mathrm{eV}\right)$ among all other structures formed during spontaneous adsorption of a silicon atom on $\mathrm{Si}(100)-2 \times 1-\mathrm{Cl}$.

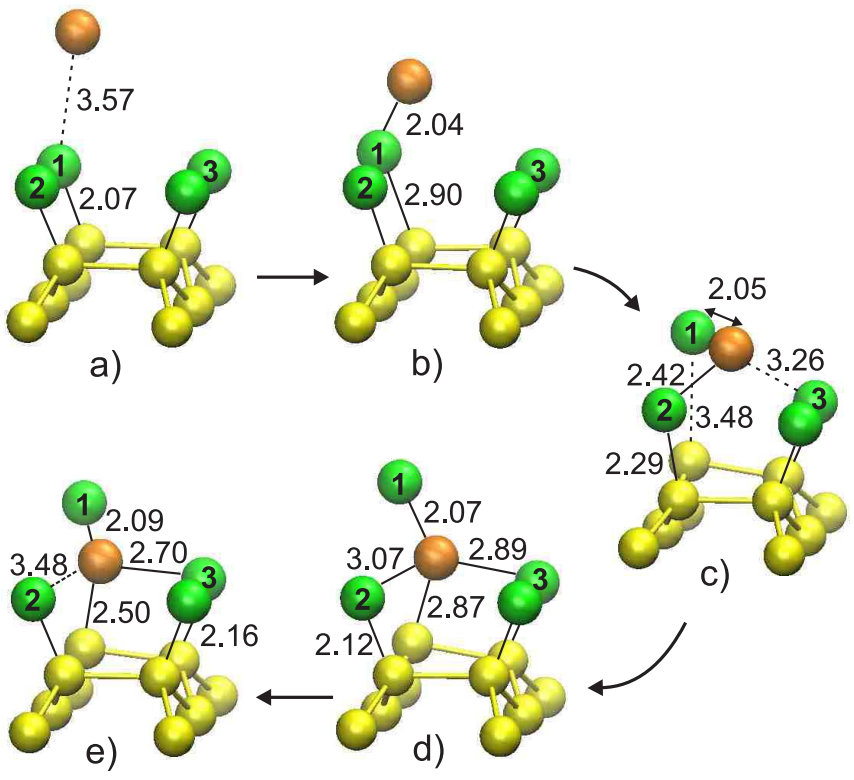

Figure 2. The process of geometry optimization for spontaneous substitutional adsorption. a) Initial structure; b-d) three structures through which the process of coordinates relaxation passes; e) optimized structure E.

Since such substitutional adsorption turned out to be very stable, we have considered additional adsorption positions of a silicon atom under the chlorine monolayer, which could be obtained by $\mathrm{Si}_{a d}$ migration from its position in the structure $\mathrm{E}$. The structure $\mathrm{F}$ is similar to $\mathrm{E}$, but the position of $\mathrm{Si}_{a d}$ is shifted closer to the row between the dimers. The adsorption energies of the structures $\mathrm{E}$ and $\mathrm{F}$ containing a $\mathrm{SiCl}$ cluster are approximately equal each other $(-2.83 \mathrm{eV}$ and $-2.89 \mathrm{eV}$, respectively).

In the most favorable configurations, $\mathrm{Si}_{a d}$ forms $\mathrm{SiCl}_{2}$ clusters in the inter-bridge and bridge dimer sites (structures $\mathrm{G}$ and $\mathrm{H}$ in Fig. (1). Atomic configuration G appears to be the most stable structure of all considered in this paper. This result strongly suggests that the formation of the $\mathrm{Si}_{a d}-\mathrm{Si}_{s}$ bonds makes a valuable contribution to the lowering of the adsorption energy in comparison with the formation of $\mathrm{Si}_{a d}-\mathrm{Cl}$ bonds only. Note that $\mathrm{Si}-\mathrm{Si}$ surface dimer in model $\mathrm{H}$ is not tilted, while in models $\mathrm{G}$ and $\mathrm{E}$ it is slightly tilted by $5^{\circ}$ and $4^{\circ}$, respectively.

Transitions from the structure $\mathrm{F}$ to the most stable structures $\mathrm{G}$ and $\mathrm{H}$ are shown in the energy diagram in Fig. 3. The $\mathrm{SiCl}$ cluster in structure $\mathrm{F}$ can attach the chlorine atom $\mathrm{Cl}(3)$ from the nearest dimer and migrate to the most stable inter-bridge dimer site (structure G). To break the bond between $\mathrm{Cl}(3)$ and the surface atom 


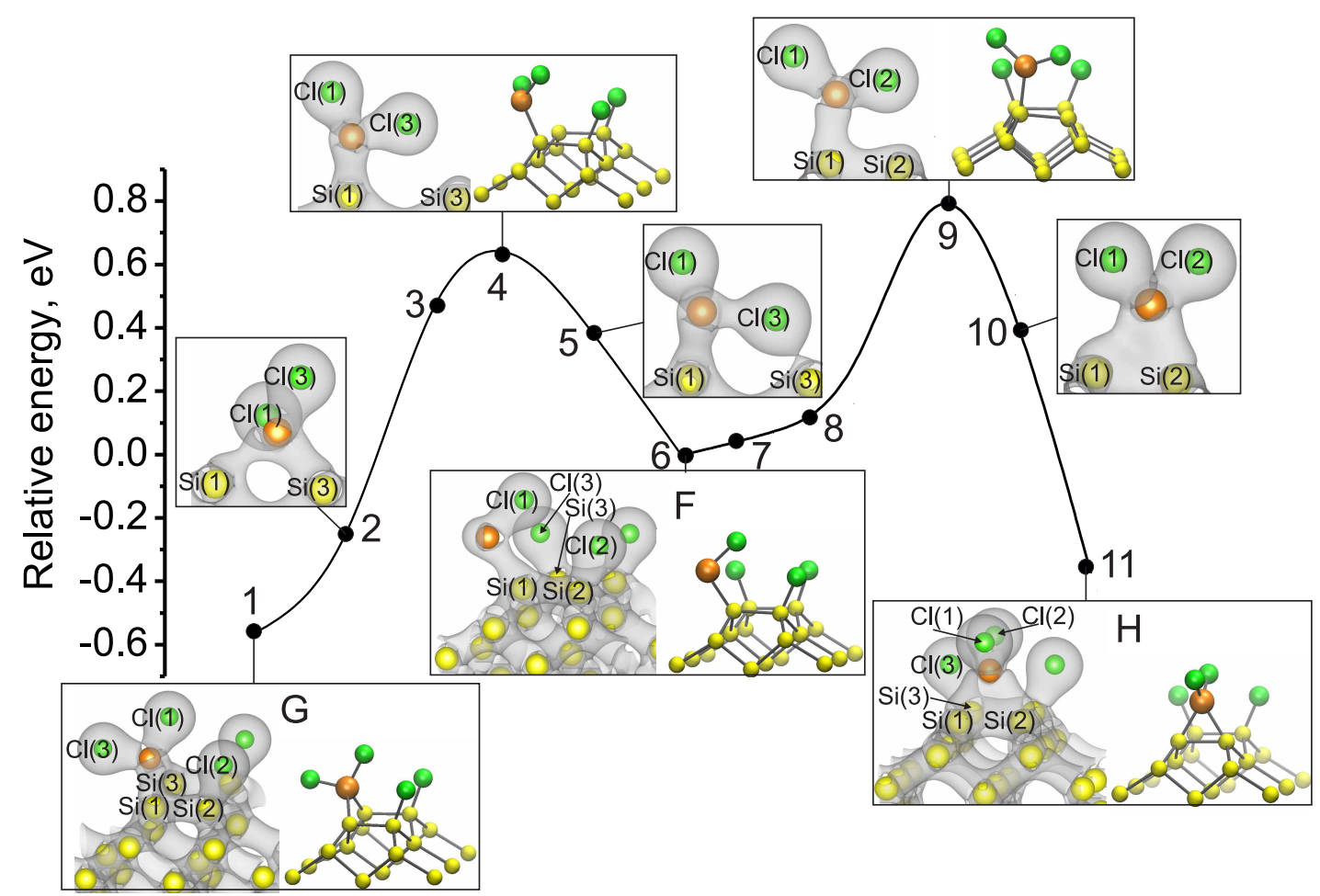

Figure 3. Reaction pathway for transitions from the structure F (image 6) to the most stable structures G (image 1) and $\mathrm{H}$ (image 11).Charge density distribution is shown for initial, final, and some intermediate states.

$\mathrm{Si}_{s}(3)$, an energy of $0.64 \mathrm{eV}$ is required. The charge density distribution in Fig. 3 3 clearly shows $\mathrm{Cl}(3)-\mathrm{Si}_{s}(3)$ bond breaking (inserts $5 \rightarrow 4$ ) and $\mathrm{Si}_{a d}-\mathrm{Si}_{s}(3)$ bond formation (inserts $4 \rightarrow 2)$. The reverse pathway $(\mathrm{G} \rightarrow \mathrm{F})$ requires a higher activation energy, since a stronger bond between $\mathrm{Si}_{a d}$ and $\mathrm{Si}_{s}$ should be broken. The transformation of the $\mathrm{SiCl}$ cluster in the structure $\mathrm{F}$ to the $\mathrm{SiCl}_{2}$ cluster in the bridge dimer site (structure $\mathrm{H}$ ) is an alternative process. This process is not energetically preferable, since the structure $\mathrm{H}$ is less favorable by $0.20 \mathrm{eV}$ and the activation barrier for the $\mathrm{F} \rightarrow \mathrm{H}$ transition is slightly higher $(0.80 \mathrm{eV})$ than that for the $\mathrm{F} \rightarrow \mathrm{G}$ transition $(0.64 \mathrm{eV})$.

Transitions between different adsorption structures are shown in the energy diagram in Fig. 4. The $\mathrm{SiCl}_{2}^{*}$ cluster in the structure A can transfer to more stable structure B without an activation energy. Further, the structure $\mathrm{B}$ can transform to the structure $\mathrm{F}$ with low barrier $(0.06 \mathrm{eV})$. Structures $\mathrm{C}$ and $\mathrm{D}$ can also transform to the structure $\mathrm{F}$ with low barrier $(0.11$ and $0.05 \mathrm{eV}$, respectively). Pathway from the structures $\mathrm{E}$ to $\mathrm{F}$ require activation energy of $0.14 \mathrm{eV}$. Transitions $\mathrm{E} \rightarrow \mathrm{G}(\mathrm{E} \rightarrow \mathrm{H})$ requires approximately the same activation energy as transitions $\mathrm{F} \rightarrow \mathrm{G}(\mathrm{F} \rightarrow \mathrm{H})$. Thus, the simplest way from spontaneous $\mathrm{Si}_{a d}$ adsorption to the most stable adsorption site passes from the structure $\mathrm{E}$ to the structure $\mathrm{G}$ and requires an energy of $0.57 \mathrm{eV}$.

The $\mathrm{SiCl}_{2}^{*}$ cluster formed in spontaneous $\mathrm{Si}_{a d}$ adsorption can desorb as a $\mathrm{SiCl}_{2}$ molecule. Energies required for $\mathrm{SiCl}_{2}$ molecules desorption $\left(E_{\text {des }}\right)$, as well as energy difference between final $\left(E_{f}\right)$ and initial $\left(E_{i}\right)$ states, are listed in Table [I] The process of $\mathrm{SiCl}_{2}$ desorption from the structure $\mathrm{A}$ is exothermic and it can occur over a very low barrier $\left(E_{a c t}=0.01 \mathrm{eV}\right)$ (Fig. 4). In the structure B, a $\mathrm{SiCl}_{2}$ molecule desorbs over a rather low barrier. Therefore, as soon as a $\mathrm{SiCl}_{2}^{*}$ cluster forms on the surface, it can desorb as a $\mathrm{SiCl}_{2}$ molecule. The $\mathrm{SiCl}_{2}$ molecule desorption from the structures $\mathrm{C}$ and $\mathrm{D}$ requires higher activation barrier, $0.38 \mathrm{eV}$ and $1.38 \mathrm{eV}$, correspondingly. Note that desorption of $\mathrm{SiCl}_{3}\left(\mathrm{SiCl}_{4}\right)$ from the structure C (D) is less beneficial than the desorption of $\mathrm{SiCl}_{2}$ molecule.

Table II. Energy difference between final $\left(E_{f}=-1.39 \mathrm{eV}\right)$ and initial $\left(E_{i}\right)$ states and energy barrier required for desorption. In the final state, a $\mathrm{SiCl}_{2}$ molecule is above the surface, and a double $\mathrm{Cl}$ vacancy (on one dimer) is formed on the surface. In the case of desorption process without activation energy, energy barrier coincides with the energy difference $\left(E_{f}-E_{i}\right)$.

\begin{tabular}{|c|c|c|}
\hline Model & $\left(E_{f}-E_{i}\right), \mathrm{eV}$ & Energy barrier, eV \\
\hline \hline $\mathrm{A}$ & -0.64 & 0.01 \\
\hline $\mathrm{B}$ & 0.12 & 0.12 \\
\hline $\mathrm{C}$ & 0.38 & 0.38 \\
\hline $\mathrm{D}$ & 0.02 & 1.38 \\
\hline
\end{tabular}




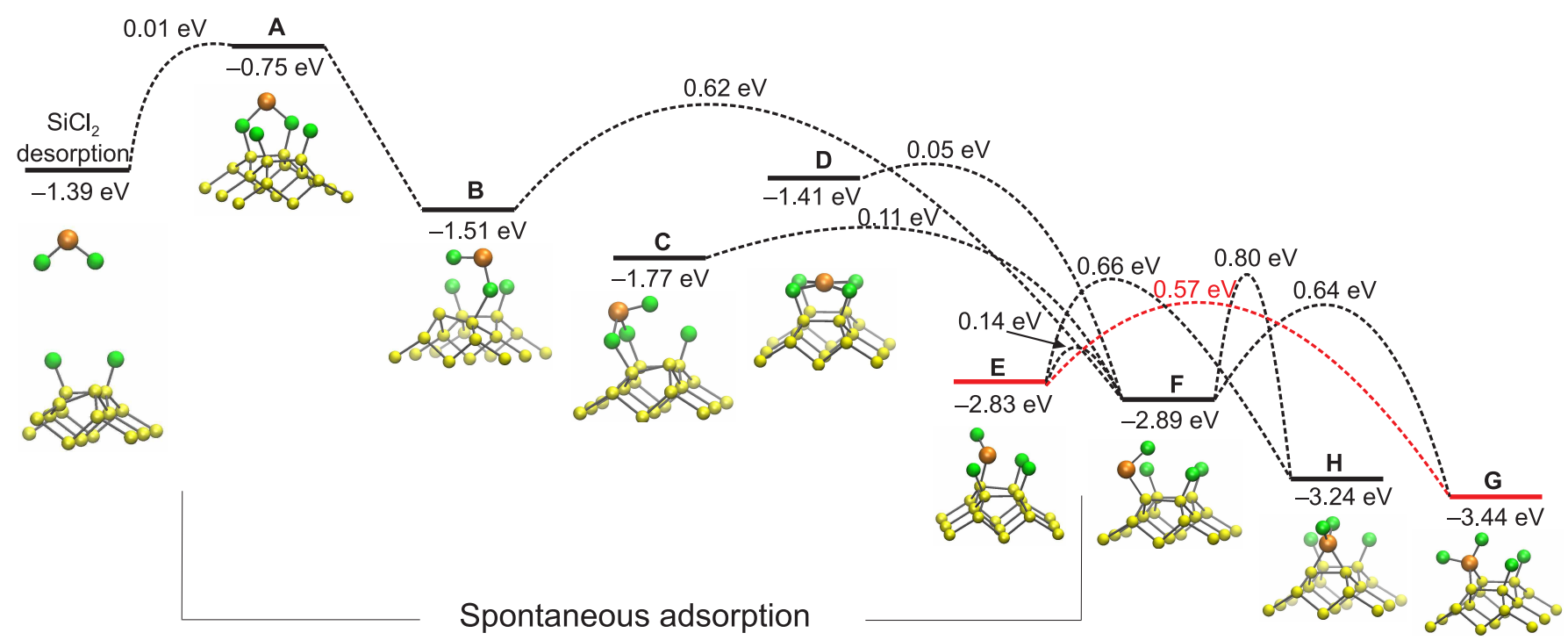

Figure 4. Transitions between adsorption structures $\mathrm{A}-\mathrm{G}$ for $\mathrm{Si}_{a d}$ on $\mathrm{Si}(100)-2 \times 1-\mathrm{Cl}$. The simplest way from spontaneous $\mathrm{Si}_{a d}$ adsorption to the most stable adsorption site is marked by the red curve. Silicon atoms are yellow, chlorine atoms are green, and $\mathrm{Si}$ adatom is orange.

\section{DISCUSSION}

In this section, we turn to the comparison of Si adsorption on a Cl-terminated $\operatorname{Si}(100)-2 \times 1$ surface with a H-terminated one ${ }^{12}$. In both cases, the Si adatom, spontaneously substituting $\mathrm{Z}(\mathrm{Z}=\mathrm{H}, \mathrm{Cl})$ atom, can capture one more $\mathrm{Z}$ atom and form a $\mathrm{SiZ}_{2}$ cluster in a bridge dimer or an inter-bridge dimer site.

In the case of $\mathrm{Si}$ adsorption on hydrogenated surface at room temperature, most of $\mathrm{Si}$ adatoms transfer from the substitutional site to the bridge dimer site due to the low activation energy $(0.5 \mathrm{eV})^{14}$. Further transition of the $\mathrm{SiH}_{2}$ cluster into the most favorable position in the inter-bridge dimer site requires an activation energy of $1.1 \mathrm{eV}^{14}$, which can hardly be overcome at room temperature. This scenario is in agreement with the experiment ${ }^{14}$, where $\mathrm{Si}$ adatoms deposited on a H-terminated $\mathrm{Si}(100)-2 \times 1$ surface at room temperature were at first found predominantly in the bridge dimer sites, but after annealing to $250^{\circ} \mathrm{C}$ - in the inter-bridge dimer sites.

According to our calculations of chlorinated surface, the minimum energy path from the structure E to structure $\mathrm{H}$ requires an activation energy of $0.66 \mathrm{eV}$, whereas the transition from the structure $\mathrm{E}$ to the most stable structure $(\mathrm{G})$ requires only $0.57 \mathrm{eV}$. Thus, most $\mathrm{Si}$ adatoms deposited on a $\mathrm{Si}(100)-2 \times 1-\mathrm{Cl}$ surface at room temperature should be adsorbed into the inter-bridge dimer site. According to the silicon crystal structure, the right position for the growth of a new layer is the bridge dimer site but not the inter-bridge dimer site. However, at $250^{\circ} \mathrm{C}$, Si adatoms adsorbed on $\mathrm{Si}(100)-2 \times 1-\mathrm{H}$ in the inter-bridge dimer sites do form a silicon crystal structure (according to transmission electron microscopy (TEM) experiments $\left.\frac{8,10}{10}\right)$. To explain the successful formation of a new crystalline layer from adatoms at the inter-bridge dimer sites, it is necessary to study the process with two or more Si adatoms.

We have found that the most stable structures are the same for Si adsorbed on $\mathrm{H}$ - and Cl-terminated $\mathrm{Si}(100)$ $2 \times 1$ and also the activation barriers are comparable, so we can expect that the epitaxy process will be similar. In the case of $\mathrm{H}$-terminated $\mathrm{Si}(100)-2 \times 1$, it has been experimentally shown that silicon epitaxy on hydrogen monolayer leads to hydrogen segregation to the surface ${ }^{8}$. Therefore it proves the possibility of Si overgrowth on chlorine monolayer with $\mathrm{Cl}$ segregation to the surface without $\mathrm{Cl}$ embedding in the epitaxial layers.

The difference between $\mathrm{Si}$ adsorption on $\mathrm{Si}(100)-2 \times 1-\mathrm{H}$ and $\mathrm{Si}(100)-2 \times 1-\mathrm{Cl}$ is that in the later case, $\mathrm{SiCl}_{2}^{*}$ clusters weakly bound to the Cl-terminated surface are formed. $\mathrm{A} \mathrm{SiCl}_{2}$ molecule can easily desorb from structures $\mathrm{A}$ and $\mathrm{B}$ containing $\mathrm{SiCl}_{2}^{*}$ clusters, since the energy barriers appear to be very low. As $\mathrm{SiCl}_{2}$ desorbs, chlorine is also partly removed from a $\mathrm{Si}(100)-2 \times 1-\mathrm{Cl}$ surface. In other words, the adsorbed silicon atoms etch the chlorine layer but do not remove Si substrate atoms. The subsequent Si overgrowth on the substrate area free of $\mathrm{Cl}$ should lead to a more uniform growth of the epitaxial layers due to increased adatom mobility on $\mathrm{Cl}$-free surface (for example, $\mathrm{Si}$ adatom mobility is lower on $\mathrm{Si}(100)-2 \times 1$ surfaces terminated by hydrogen ${ }^{11}$ and bromine ${ }^{32}$ than on a clean surface). Note that the low-temperature removal of $\mathrm{Cl}$ will not lead to the diffusion of dopants (for example, $\mathrm{P}$ atoms) on the surface. 


\section{CONCLUSIONS}

The structures and energetics of $\mathrm{Si}$ adsorption on $\mathrm{Si}(100)-2 \times 1-\mathrm{Cl}$ have been studied with the density function theory. The activation barriers for transitions between the most stable states and desorption of $\mathrm{SiCl}_{2}$ molecules from the surface have also been calculated. When compared to the common practice of using a hydrogen monolayer as a resist, a chlorine monolayer presents not just similarities, but also potential advantages due to low-temperature removal of chlorine during Si epitaxy. Firstly, spontaneous substitutional adsorption takes place both on $\mathrm{H}-14$ and Cl-terminated $\mathrm{Si}(100)$ $2 \times 1$. Secondly, the most stable sites for $\mathrm{Si}_{a d}$ on both $\mathrm{H}$ 14 and $\mathrm{Cl}$-terminated surfaces are inter-bridge and bridge dimer sites, where $\mathrm{Si}_{a d}$ is bound to two $\mathrm{Si}$ and two $\mathrm{H}$ (or $\mathrm{Cl}$ ) atoms. These results indicate that chlorine segregates to the surface during Si deposition and is not incorporated into the epitaxial layers. Thirdly, we have found out that $\mathrm{SiCl}_{2}^{*}$ clusters formed during silicon adsorption can easily desorb from a Cl-terminated surface. Thus, depositing silicon on a chlorine monolayer should produce silicon epitaxial layers of quality at least not worse (may be even better) than on a hydrogen monolayer.

\section{ACKNOWLEDGEMENT}

The work was supported by Russian Science Foundation (grant 16-12-00050). We also thank the Joint Supercomputer Center of RAS for providing the computing power.

* pavlova@kapella.gpi.ru

1 T.-C. Shen, J.-Y. Ji, M. A. Zudov, R.-R. Du, J. S. Kline, and J. R. Tucker, Appl. Phys. Lett. 80, 1580 (2002).

2 J. Keizer, S. Koelling, P. Koenraad, and M. Simmons, ACS Nano 9, 2537 (2015).

3 B. Weber, S. Mahapatra, H. Ryu, S. Lee, A. Fuhrer, T. Reusch, D. L. Thompson, W. C. T. Lee, G. Klimeck, L. C. L. Hollenberg, and M. Simmons, Science 335, 64 (2012).

4 B. Weber, H. Ryu, Y.-H. M. Tan, G. Klimeck, and M. Y. Simmons, Phys. Rev. Lett. 113, 246802 (2014)

5 M. Fuechsle, J. Miwa, S. Mahapatra, H. Ryu, S. Lee, O. Warschkow, L. C L Hollenberg, G. Klimeck, and M. Simmons, Nat. Nanotechnol. 7, 242 (2012).

${ }^{6}$ L. Oberbeck, N. J. Curson, M. Y. Simmons, R. Brenner, A. R. Hamilton, S. R. Schofield, and R. G. Clark, Appl. Phys. Lett. 81, 3197 (2002).

7 M. A. Broome, S. Gorman, M. House, S. Hile, J. Keizer, D. Keith, C. D. Hill, T. Watson, W. Baker, L. C. L. Hollenberg, and M. Simmons, Nat. Commun. 9, 980 (2018).

8 X. Deng, P. Namboodiri, K. Li, X. Wang, G. Stan, A. F. Myers, X. Cheng, T. Li, and R. M. Silver, Appl. Surf. Sci. 378, 301 (2016)

9 J. A. Hagmann, X. Wang, P. Namboodiri, J. Wyrick, R. Murray, M. D. Stewart, R. M. Silver, and C. A. Richter, Appl. Phys. Lett. 112, 043102 (2018)

10 X. Wang, J. A. Hagmann, P. Namboodiri, J. Wyrick, K. Li, R. E. Murray, A. Myers, F. Misenkosen, M. D. Stewart, C. A. Richter, and R. M. Silver, Nanoscale 10, 4488 (2018).

11 J. Nara, T. Sasaki, Phys. Rev. Lett. 79, 4421 (1997)

12 S. Jeong and Phys. Rev. Lett. 79, 4425 (1997).

13 S. Jeong and Phys. Rev. B 58, 12958 (1998) and T. Ohno,

A. Oshiyama,

A. Oshiyama,

J. Phys. Soc. Jpn. 74, 389 (2005).

15 J.-Y. Ji and T.-C. Shen, Phys. Rev. B 70, 115309 (2004).

16 S. Moon, C. Jeon, H. Hwang, C. Hwang, H. Song, H. Shin, S. Chung, and C. Park, Adv. Mater. 19, 1321 (2007).

17 C. Jeon, H.-N. Hwang, H.-J. Shin, C.-Y. Park, and C.-C. Hwang, Appl. Surf. Sci. 257, 8794 (2011).

18 G. A. de Wijs, A. De Vita, and A. Selloni, Phys. Rev. B 57, 10021 (1998).

19 C. M. Aldao, A. Agrawal, R. E. Butera, and J. H. Weaver, Phys. Rev. B 79, 125303 (2009).

20 T. V. Pavlova, G. M. Zhidomirov, and K. N. Eltsov, J. Phys. Chem. C 122, 1741 (2018)

21 M. A. Hall, C. Mui, and C. B. Musgrave, J. Phys. Chem. B 105, 12068 (2001)

22 N. Kunioshi, Y. Fujimura, A. Fuwa, and K. Yamaguchi, Comp. Mater. Sci. 155, 28 (2018).

23 S. Yadav and C. V. Singh, \begin{tabular}{llll}
\hline Appl. Surf. Sci. 475, $124(2019)$ & \\
F. & Gao & and & A. V. Teplyakov,
\end{tabular} $\begin{array}{lll} & \\ \text { J. Phys. Chem. C 118, } 27998(2014) \text {. Teplyakov, }\end{array}$

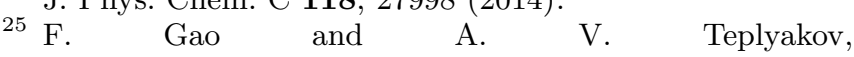
J. Phys. Chem. C 120, 5539 (2016).

26 B. Lange and W. Schmidt, Surf. Sci. 602, 1207 (2008).

27 F. A. Soria, E. M. Patrito, and P. Paredes-Olivera, J. Phys. Chem. C 117, 18021 (2013).

28 G. Kresse and J. Hafner, Phys. Rev. B 47, 558 (1993)

29 G. Kresse and J. Furthmüller, Phys. Rev. B 54, 11169 (1996).

30 J. P. Perdew, K. Burke, and M. Ernzerhof, Phys. Rev. Lett. 77, 3865 (1996).

31 G. Henkelman, B. P. Uberuaga, and H. Jónsson, J. Chem. Phys. 113, 9901 (2000)

32 G. J. Xu and J. H. Weaver, Phys. Rev. B 70, 165321 (2004)

14 H. Kajiyama, Y. Suwa, S. Heike, M. Fujimori, J. Nara, T. Ohno, S. Matsuura, T. Hitosugi, and T. Hashizume, 